judgements of life satisfaction coupled with an absence of negative feelings. Little is known about levels of well-being in the context of adaptation to chronic illness. This paper will explore the impact of chronic illness on the well-being of family members.

Methods Data come from wave 1 of Understanding Society, a new longitudinal UK-representative household panel survey. Subjective well-being of adults (16 years) was measured using the GHO-12, the Warwick-Edinburgh Mental Well-being Scale and a question on life satisfaction. The Strengths and Difficulties Questionnaire measured well-being in youth (aged 10-15 years). Selfreported long-term limiting illness (LLTI) was used to indicate chronic illness. Latent variable models were used to explore associations between partners $(\mathrm{N}=4167)$ and among family members $(\mathrm{n}=3056)$.

Results LLTI in one member of a cohabiting partnership was negatively associated not only with their own well-being but also that of their partner. There were no differences by gender. The association between a parent's LLTI and their adult child's well-being was not significant, regardless of the gender of the parent. There was a significant association between mother's LLTI and SDO total difficulties score for younger children; however no association was observed for father's Illness.

Conclusions There are associations between one's own illness on both themselves and their partners. A parent's LLTI was not associated with worse well-being in adult children but was associated with increased difficulties in their younger children.

\section{P2-31 ASSOCIATION BETWEEN PLASMA VITAMIN D AND METABOLIC SYNDROME IN THE CANADIAN POPULATION}

doi:10.1136/jech.2011.142976h.67

${ }^{1,2} \mathrm{D}$ Brenner, * 1,2P Arora, ${ }^{2,3} \mathrm{~B}$ Garcia-Bailo, ${ }^{3} \mathrm{~T}$ Wolever, ${ }^{3} \mathrm{~A}$ El-Sohemy, ${ }^{2,3} \mathrm{M}$ Karmali, ${ }^{2}$ A Badawi. 'Dalla Lana School of Public Health, University of Toronto, Toronto, Ontario, Canada; ${ }^{2}$ Office of Biotechnology Genomics and PublOffice of Biotechnology, Genomics and Public Health, Public Health Agency of, Toronto, Ontario, Canada; ${ }^{3}$ Department of Nutritional Sciences, University of Toronto, Toronto, Ontario, Canada

Background Vitamin D deficiency has been implicated in the susceptibility to the metabolic syndrome and a spectrum of conditions, such as obesity and type 2 diabetes mellitus. The present study aimed to quantify the association between vitamin $\mathrm{D}$ serum levels and the occurrence of metabolic syndrome components and insulin resistance among Canadian adults.

Methods Vitamin D serum levels and the related clinical data were extracted from 1920 subjects from the Canadian Health Measures Survey, a national survey representing the general Canadian population. The definition of the metabolic syndrome components was based on the National Cholesterol Education Program, Adult Treatment Panel III criteria. Adjusted unconditional logistic regression models were used to estimate the association between vitamin $\mathrm{D}$ level quartiles and risk of having metabolic syndrome, as well as the association between plasma vitamin $\mathrm{D}$ and insulin resistance (HOMA-IR).

Results Within the survey, $11.4 \%$ of the subjects had the metabolic syndrome. Increasing levels of plasma vitamin $\mathrm{D}$ were positively correlated with reduced numbers of metabolic syndrome components. Subjects in the highest quartile had significantly lower risk of having metabolic syndrome compared to those in the lowest vitamin $\mathrm{D}$ quartile ( $\mathrm{OR}=0.36,95 \%$ CI 0.19 to 0.66 ). Furthermore, increasing plasma vitamin $\mathrm{D}$ levels were associated with lower HOMA-IR scores $(\beta=-0.88, p=0.004)$ in a fully adjusted linear model.

Conclusion Vitamin D serum levels can predict the occurrence of metabolic syndrome and insulin resistance among Canadian adults.

\section{P2-32 VITAMIN D IN THE PREDICTION OF METABOLIC SYNDROME: A TARGET FOR PUBLIC HEALTH INTERVENTION}

doi:10.1136/jech.2011.142976h.68

${ }^{1,2} \mathrm{D}$ Brenner, ${ }^{* 1,2} \mathrm{P}$ Arora, ${ }^{2,3} \mathrm{~B}$ Garica-Bailo, ${ }^{3} \mathrm{~A}$ El-Sohemy, ${ }^{2,3} \mathrm{M}$ Karmali, ${ }^{2} \mathrm{~A}$ Badawi. ${ }^{1}$ Dalla Lana School of Public Health, University of Toronto, Toronto, Ontario, Canada: ${ }^{2}$ Office of Biotechnology Genomics and Public Health, Public Health Agency of Canada, Toronto, Ontario, Canada; ${ }^{3}$ Department of Nutritional Sciences, University of Toronto, Toronto, Ontario, Canada

Background The association between vitamin $\mathrm{D}(\mathrm{VitD})$ and cardiometabolic health has been described however the ability of VitD to predict metabolic syndrome (MetSyn) beyond individual demographics and common lifestyle factors is unclear.

Methods 1799 adults from the Canadian Health Measures Survey, a nationally representative survey were examined. MetSyn was defined based on the National Cholesterol Education Program's Adult Treatment Panel III criteria. The comparative value of smoking status, energy expenditure from physical activity and plasma $25(\mathrm{OH}) \mathrm{D}$ VitD to predict the presence of metabolic syndrome was evaluated using receiver operating characteristic curves (ROC) from fully adjusted logistic regression models (age, sex, ethnicity, education, annual fruit intake and month of interview). Somer's D and $c$ statistics were used to compare across models. Sampling weights were applied to all models.

Results Inclusion of plasma VitD, energy expenditure and smoking status all lead to increases in the $c$ statistic and Somer's D suggesting increased predictive ability compared to the baseline model. ROC contrasts, however, showed that only inclusion of $\mathrm{VitD}$ significantly increased the predictive ability of the model $(p=0.001)$.

Discussion VitD may be a useful predictive tool for cardiometabolic risk prediction in addition to conventional factors. Prospective analyses may provide more insight into $\mathrm{VitD}$ as a potential population-based intervention target for reduction of chronic disease burden related to MetSyn.

\section{P2-33 THE IMPACT OF THE METABOLIC SYNDROME ON CARDIOMETABOLIC AND INFLAMMATORY PROFILES AMONG CANADIAN ADULTS}

doi:10.1136/jech.2011.142976h.69

${ }^{1,2}$ D Brenner, ${ }^{*} 1,2 \mathrm{P}$ Arora, ${ }^{2} \mathrm{M}$ Karmali, ${ }^{2} \mathrm{~A}$ Badawi. ${ }^{1}$ Dalla Lana School of Public Health, University of Toronto, Toronto, Ontario, Canada; ${ }^{2}$ Office of Biotechnology Genomics and Public Health, Public Health Agency of Canada, Toronto, Ontario, Canada

Background The metabolic syndrome (MetSyn) is known as a precursor condition for a spectrum of cardiometabolic complications including type 2 diabetes and cardiovascular disease. The present analysis aimed to quantify the differences in serum levels of cardiometabolic and inflammatory markers across the number of MetSyn components among Canadian adults.

Methods Serum levels of apolipoprotein A1 and B (ApoA1, B), creatine, total cholesterol/HDL cholesterol ratio (TC:HDL), C reactive protein (CRP), fibrinogen, glycosylated haemoglobin (HbA1c) and homocysteine were extracted from 1920 adults from the Canadian Health Measures Survey (CHMS). The definition of MetSyn components was based on the National Cholesterol Education Program, Adult Treatment Panel III criteria. Generalised linear models adjusted for age, sex, physical activity, smoking and ethnicity were used to quantify the relationship between select markers and number of MetSyn components

Results Among survey subjects, 11.4\% had MetSyn with $59.6 \%$ having at least one component. We observed several significant relationships between markers with increasing numbers of MetSyn components. Mean levels of ApoB, creatine, (TC:HDL), CRP, 
fibrinogen, HbA1c increased significantly as the numbers of MetSyn components increased whereas levels of ApoA1 decreased $(p<0.05)$. Conclusions Our results suggest that increasing numbers of metabolic syndrome components are associated with an elevated level of both markers of chronic low-grade inflammation and intermediate disease. Our findings support previous work showing that persons with MetSyn are a clinically relevant population with underlying pathogenesis that could benefit from early treatment.

\section{P2-34 ASSOCIATION OF ANTHROPOMETRIC AND LIFESTYLE FACTORS WITH PROSTATE SPECIFIC ANTIGEN (PSA) TRAJECTORIES IN MEN WITH LOCALISED PROSTATE CANCER UNDERGOING ACTIVE MONITORING}

doi:10.1136/jech.2011.142976h.70

${ }^{1} \mathrm{~A}$ Burton, ${ }^{* 1} \mathrm{R}$ Martin, ${ }^{1} \mathrm{~J}$ Holly, ${ }^{2} \mathrm{~F}$ Hamdy, ${ }^{3} \mathrm{D}$ Neal, ${ }^{1} \mathrm{~J}$ Donovan, ${ }^{1} \mathrm{~K}$ Tilling. ${ }^{1}$ University of Bristol, Bristol, UK; ${ }^{2}$ University of Oxford, Oxford, UK; ${ }^{3}$ University of Cambridge, Cambridge, UK

Introduction Widespread use of Prostate-specific Antigen (PSA) testing has lead to a rapid increase in the identification of low risk prostate cancer, with PSA trajectories often used to monitor tumour progression after diagnosis. Here we develop novel age-specific multi-level growth curves to assess the effects of lifestyle and anthropometric measures on PSA trajectories.

Methods Serial PSA measures from 513 men aged $50-70$ years with localised prostate cancer undergoing active monitoring were used to develop models. Gleason score, height, weight, body mass index, waist, waist to hip ratio, smoking and alcohol consumption were each added to the basic age-specific growth curve model as explanatory variables.

Results The basic growth curve gave an average PSA at age 50 of $2.10 \mathrm{ng} / \mathrm{ml}$ (95\% CI 1.85 to 2.38 ) and a yearly increase in PSA of $1.07 \mathrm{ng} / \mathrm{ml}$ (95\% CI 1.06 to 1.08 ) (or 7\%; 95\% CI 6\% to 8\%). In these preliminary findings, Gleason score at baseline was strongly associated with PSA growth: the yearly increase in PSA was 3.4\% greater ( $95 \%$ CI $1 \%$ to $6 \%$ ) for men with Gleason of 7 or greater vs those with Gleason 6 or less. Current smoking was positively associated with PSA change: the yearly increase in PSA was $3.2 \%$ greater $(95 \%$ CI $0 \%$ to $7 \%$ ) for current vs never smokers. No other factors were strongly associated with either initial PSA or yearly increase in PSA. Conclusions Smoking status may be associated with PSA trajectory in men being followed up by active monitoring but other lifestyle and anthropometric factors have little association.

\section{P2-35 OUTCOMES OF SMALL FOR GESTATIONAL AGE (SGA) BIRTH AS A PLACENTA ASSOCIATED SYNDROME}

doi:10.1136/jech.2011.142976h.71

\begin{abstract}
1,2M K Campbell, * 1,2W R Avison, 1,2K N Speechley. 'Schulich School of Medicine and Dentistry, The University of Western Ontario, London, Ontario, Canada; ${ }^{2}$ The Children's Health Research Institute, London, Ontario, Canada
\end{abstract}

Introduction Infants born small for gestational age (SGA) are at higher risk for morbidity. This analysis explored whether increased risk is attributable to SGA or its causes.

Methods A population-based cohort $(n=2357)$ was recruited at 10-22 weeks gestation. We collected prenatal data by questionnaire, perinatal data from hospital records, and follow-up data by telephone survey 2-4 years later. Outcomes included neonatal admission to special care nursery (NASC), neonatal Apgar score $<7$ at 1 min (Apgar1) and, hospital admission (HA) during the 12 months preceding the follow-up interview. Analyses included multivariable logistic regression.
Results Preeclampsia had statistically significant multivariable associations with NASC and Apgar1; OR's (95\% CI's) were 2.0 (1.0 to 5.0 ) and 2.4 (95\% CI 1.3 to 4.3), respectively. Apgar1 was also associated with young maternal age and obesity. In secondary analysis restricted to term deliveries, threatened preterm labour (TPL) had OR's of $3.9(1.6,9.3)$ and $2.2(1.2,4.2)$, respectively, for NASC and Apgar1. TPL displaced preeclampsia from the model indicating correlation. Since recent literature suggests that preeclampsia, TPL, and SGA have common aetiologies, we combined these into a single construct, Placenta Associated Syndromes (PAS) (Alilu et al 2010). The OR's of PAS with NSC and Apgar1 were 3.9 $(1.6,9.3)$ and $2.2(1.2,4.2)$, respectively. HA had multivariable associations with preeclampsia and maternal depression (CESD $>24)$ with OR's of $2.6(1.1,6.1)$ and $2.9(1.3,6.4)$. SGA was not independently associated with outcomes studied.

Conclusion We conclude that SGA is a marker for placental dysfunction, which is the true risk factor for the outcomes studied.

\section{P2-36 BODY MASS INDEX AND RISK OF INCIDENT ISCHAEMIC HEART DISEASE IN WOMEN: A PROSPECTIVE COHORT STUDY}

doi:10.1136/jech.2011.142976h.72

D Canoy, ${ }^{*}$ B J Cairns, A Balkwill, G K Reeves, J Green, V Beral. Cancer Epidemiology Unit, University of Oxford, Oxford, UK

Background Various studies have examined body mass index in relation to mortality due to ischaemic heart disease (IHD), but few data are available on incident IHD including non-fatal events particularly in women.

Methods We used multivariate Cox regression, taking into account confounding and mediating factors, to examine the association between body mass index and first IHD event (ascertained from hospital records and death registration) in the Million Women Study, a population-based prospective cohort of middle age British women.

Results In this cohort of women aged $50-70$ years, there were 48895 incident IHD events during 10.7 million person-years of follow-up, including 5108 deaths with IHD identified as the primary cause. IHD incidence rate was 2.1 per 100 women per 5 years. Mean measured BMI (SD) was $26.7(4.7) \mathrm{kg} / \mathrm{m}^{2}$. Risk for IHD increased with higher BMI $(p<0.001)$. The large number of incident IHD events in this cohort makes it possible to examine in detail possible effect modification by other risk factors, including comparing associations between current and never smokers, or by socioeconomic status or physical activity level.

Conclusion Non-fatal IHD adds considerably to the burden of heart disease. In this very large prospective study, incident IHD in women was strongly associated with excess weight.

\section{P2-37 ASSOCIATION BETWEEN SLEEP DURATION AND ALL- CAUSE MORTALITY IN OLD AGE: 9-YEAR FOLLOW-UP OF THE BAMBUÍ COHORT STUDY, BRAZIL}

doi:10.1136/jech.2011.142976h.73

\begin{abstract}
${ }^{1,2}{ }^{2}$ Castro-Costa, ${ }^{* 2} \mathrm{M}$ Dewey, ${ }^{2} \mathrm{C}$ Ferri, ${ }^{1,3} \mathrm{E}$ Uchoa, ${ }^{1} \mathrm{~J}$ Firmo, ${ }^{4} \mathrm{~F}$ Rocha, ${ }^{2} \mathrm{M}$ Prince ${ }^{1,3} \mathrm{M}$ F Lima-Costa, ${ }^{2} \mathrm{R}$ Stewart. ${ }^{1}$ Centro de Pesquisa Rene Rachou, Fiocruz, Belo Horizonte, Minas Gerais, Brazil; ${ }^{2}$ King's College London, Institute of Psychiatry, London, UK; ${ }^{3}$ Federal University of Minas Gerais Medical School, Belo Horizonte, Minas Gerais, Brazil; " IPSEMG, Belo Horizonte, Minas Gerais, Brazil
\end{abstract}

Introduction This study investigates the association of sleep duration with risk of all-cause mortality among elderly Brazilians using data from a 9-year population-based cohort study. 